\title{
2D ferromagnetism in layered inorganic-organic hybrid perovskites
}

\author{
Dhani Nafday, ${ }^{1}$ Dipayan Sen $\odot,{ }^{2}$ Nitin Kaushal, ${ }^{3,4}$ Anamitra Mukherjee $\odot,{ }^{5}$ and Tanusri Saha-Dasgupta $\odot^{1,6, *}$ \\ ${ }^{1}$ Indian Association for the Cultivation of Science, $2 A \& 2 B$, Raja Subodh Chandra Mallick Road, Jadavpur, \\ Kolkata, West Bengal 700032, India \\ ${ }^{2}$ University of Calcutta, Kolkata, West Bengal 700009, India \\ ${ }^{3}$ Department of Physics and Astronomy, The University of Tennessee, Knoxville, Tennessee 37996, USA \\ ${ }^{4}$ Materials Science and Technology Division, Oak Ridge National Laboratory, Oak Ridge, Tennessee 37831, USA \\ ${ }^{5}$ School of Physical Sciences, National Institute of Science Education and Research, HBNI, Jatni 752050, India \\ ${ }^{6}$ S. N. Bose National Centre for Basic Sciences, JD Block, Sector III, Salt Lake, Kolkata, West Bengal 700106, India
}

(Received 24 August 2019; published 9 December 2019)

\begin{abstract}
Employing a combination of first-principles density functional theory and a solution of a generalized spin Hamiltonian, we predict finite-temperature ferromagnetism in a yet-to-be synthesized two-dimensional monolayer of $\mathrm{Cu}$ spins, derived out of the layered structure of inorganic-organic hybrid perovskites. The computed cleavage energies are found to be a factor of 2-3 smaller than that of graphite, thereby making the synthesis of such monolayers by exfoliation of their bulk counterparts probable. The ferromagnetic exchanges, together with in-plane magnetic anisotropy, give rise to the possibility of a finite-temperature long-range ordering of $\mathrm{Cu}$ spins, as established through the solution of a generalized model Hamiltonian. Our study should motivate future experiments in this exciting class of compounds.
\end{abstract}

DOI: 10.1103/PhysRevResearch.1.032034

Introduction. The excitement created by the synthesis of a two-dimensional (2D) allotrope of carbon, namely, graphene, by the mechanical exfoliation of the three-dimensional (3D) layered structure of graphite, has led to a surge of activity in synthesizing and predicting new functionalities in 2D materials [1]. The vast range of properties exhibited by the family of $2 \mathrm{D}$ crystals include metallicity with unprecedented mobility, topological insulators, superconductivity, charge density waves, Mott insulators, etc. [2]. However, until recently, magnetism in $2 \mathrm{D}$ crystals has not yet been realized. Long-range ferromagnetic order can be achieved in 2D crystals, and, coupled with their rich electronic properties, could lead to new magnetic and magnetoelectric applications [3].

The behavior of magnetism in low-dimensional 2D materials is subject to the Mermin-Wagner theorem [4], which states that at a finite temperature spontaneous symmetry breaking is absent in the 2D isotropic Heisenberg model. The presence of anisotropy, on the other hand, can open up gaps between the magnetically order state and the excitations, stabilizing 2D magnetism at finite temperatures. Towards this goal, making use of magnetic-field-induced anisotropy, Gong and co-workers discovered that $\mathrm{Cr}_{2} \mathrm{Ge}_{2} \mathrm{Te}_{6}$ remains ferromagnetic down to the bilayer system [5]. Huang et al. further showed that in $\mathrm{CrI}_{3}$, in which intrinsic anisotropy exists,

\footnotetext{
*t.sahadasgupta@gmail.com

Published by the American Physical Society under the terms of the Creative Commons Attribution 4.0 International license. Further distribution of this work must maintain attribution to the author(s) and the published article's title, journal citation, and DOI.
}

ferromagnetism survives down to an extreme limit of the monolayer [6-8].

This has made the entire field of 2D magnetism very topical $[9,10]$. There is, however, an urgent need to expand the list of candidate materials which would provide a better understanding of 2D magnetism in a diverse class of compounds. This calls for a critical examination of layered semiconducting systems. An important class of compounds belonging to this category are inorganic-organic layered compounds having the general formula $\left(R-\mathrm{NH}_{3}\right)_{2}\left(\mathrm{CH}_{3} \mathrm{NH}_{3}\right)_{m-1} M_{m} X_{3 m+1}$, where $R$ is an alkyl or aromatic moiety, $M$ is a metal cation, and $X$ is a halide [11]. In the extreme limit of $m=\infty$, the structure becomes a $3 \mathrm{D}$ perovskite structure with the formula $A M X_{3}$, a typical example being $\mathrm{CH}_{3} \mathrm{NH}_{3} \mathrm{PbI}_{3}$, which has been in the spotlight recently due to photovoltaic applications [12]. In the other extreme limit of $m=1$, the structure becomes layered with single layers of $M X_{4}^{2-}$ consisting of corner-shared octahedra of $M X_{6}$, separated by ordered pairs of organic ligands. To date such layered materials have been synthesized with choices of many metal cations such as $\mathrm{Cu}^{2+}, \mathrm{Ni}^{2+}, \mathrm{Mn}^{2+}$, $\mathrm{Fe}^{2+}, \mathrm{Gd}^{2+}, \mathrm{Ge}^{2+}, \mathrm{Sn}^{2+}, \mathrm{Pb}^{2+}$, etc., and halides such as $\mathrm{Cl}$, $\mathrm{Br}$, and I $[13,14]$. Use of transition-metal (TM) ions such as $\mathrm{Cu}^{2+}, \mathrm{Ni}^{2+}, \mathrm{Mn}^{2+}$, or $\mathrm{Fe}^{2+}$ brings in the prospect of magnetism. While all of them are semiconducting or insulating, most of them show superexchange-driven antiferromagnetism within the $M X_{4}{ }^{2-}$ layer with a weak interlayer magnetic exchange [15].

Among the rare examples are those of $\mathrm{Cu}^{2+}$-based systems, which are reported to show ferromagnetic ordering in the inorganic motif of the compounds, making them suitable as candidate materials to look for $2 \mathrm{D}$ ferromagnetism. The potential and prospects for these materials as $2 \mathrm{D}$ magnets need to explored. This seems to be an especially encouraging 


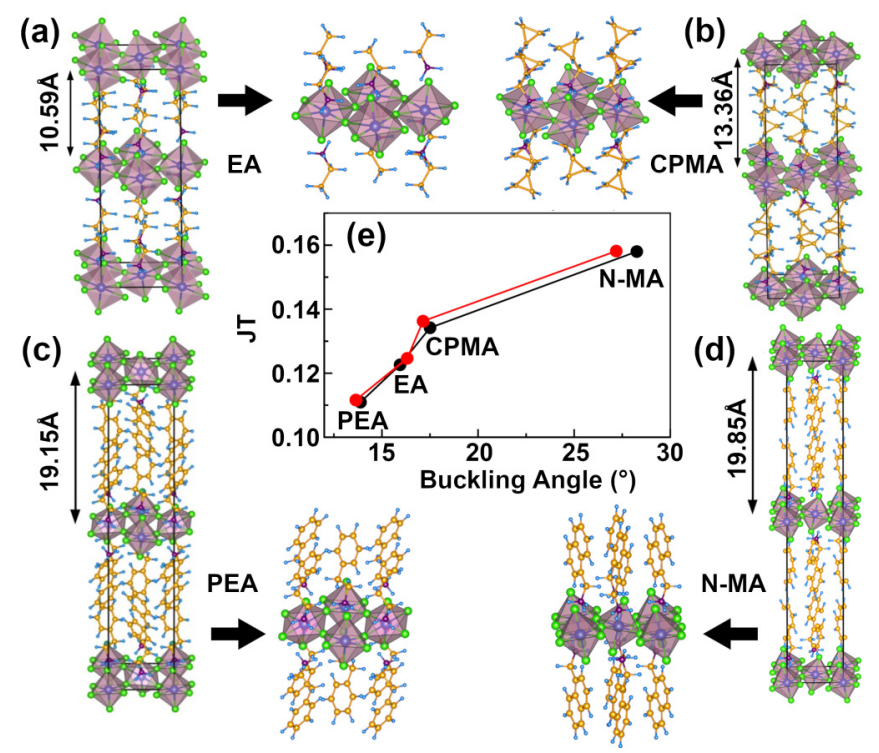

FIG. 1. Bulk crystal structures of (a) $\left(\mathrm{EA}-\mathrm{NH}_{3}\right)_{2} \mathrm{CuCl}_{4}$, (b) (CPMA- $\left.\mathrm{NH}_{3}\right)_{2} \mathrm{CuCl}_{4}$, (c) (PEA- $\left.\mathrm{NH}_{3}\right)_{2} \mathrm{CuCl}_{4}$, and (d) (Naphtha4-MA- $\left.\mathrm{NH}_{3}\right)_{2} \mathrm{CuCl}_{4}$ shown along with their $2 \mathrm{D}$ counterparts, derived out of the 3D structures. The interlayer distances in $3 \mathrm{D}$ structures are marked. (e) shows the plot of JT distortion against the buckling angle for the 2D structures (red symbols) in comparison to that of the corresponding 3D structures (black symbols).

situation given the recent report of the synthesis of atomically thin $2 \mathrm{D}$ sheets of $\left(\mathrm{C}_{4} \mathrm{H}_{9} \mathrm{NH}_{3}\right)_{2} \mathrm{PbBr}_{4},\left(\mathrm{C}_{4} \mathrm{H}_{9} \mathrm{NH}_{3}\right)_{2} \mathrm{PbCl}_{4}$, and $\left(\mathrm{C}_{4} \mathrm{H}_{9} \mathrm{NH}_{3}\right)_{2} \mathrm{PbI}_{4}[16]$.

In this Rapid Communication, we considered four $\mathrm{Cu}^{2+}$ based layered organic-inorganic complexes of a general formula $\left(R-\mathrm{NH}_{3}\right)_{2} \mathrm{CuCl}_{4}$ with $R=\mathrm{CH}_{3} \mathrm{CH}_{2}$ (EA), $\mathrm{C}_{3} \mathrm{H}_{5} \mathrm{CH}_{2}$ (CPMA), $\mathrm{C}_{6} \mathrm{H}_{5} \mathrm{CH}_{2} \mathrm{CH}_{2}$ (PEA), and $\mathrm{C}_{10} \mathrm{H}_{7} \mathrm{CH}_{2}$ (Naphtha-4MA) [17], and explored the properties of the 2D counterparts of these 3D layered compounds through ab initio density functional theory (DFT) combined with a model Hamiltonian study. Our computational exercise establishes that 2D ferromagnetism may be stabilized in a monolayer of a cornershared network of $\mathrm{CuCl}_{6}$ octahedra of these compounds, if a generalized form of anisotropy is considered. Our findings should motivate the experimental synthesis of the proposed atomically thin 2D layers and a characterization of their magnetic properties.

Crystal structure. The metal cation $(\mathrm{Cu})$ and $X$-halogen ion (Cl) form a $\mathrm{CuCl}_{6}$ octahedral unit, which corner share to form an extended inorganic layer in the structure. $\mathrm{Cu}^{2+}$ in its $d^{9}$ configuration is Jahn-Teller (JT) active, which leads to a $4+2$ coordination geometry with four short $(s)$ and two long $(l)$ $\mathrm{Cu}-\mathrm{Cl}$ bonds. In these layered compounds, the JT elongated bonds on adjacent $\mathrm{Cu}^{2+}$ ions are oriented at right angles to each other, giving rise to an antiferrodistortive arrangement.

The inorganic layers are sandwiched between organic spacers, as shown in Fig. 1. The inorganic layers are staggered with an orthorhombic space group for $\left(\mathrm{EA}-\mathrm{NH}_{3}\right)_{2} \mathrm{CuCl}_{4}$, $\left(\mathrm{CPMA}-\mathrm{NH}_{3}\right)_{2} \mathrm{CuCl}_{4}$, and $\left(\mathrm{PEA}-\mathrm{NH}_{3}\right)_{2} \mathrm{CuCl}_{4}$ [cf. Figs. 1(a)-1(c)], while they are eclipsed with a monoclinic space group for (Naphtha-4-MA-NH$\left.)_{2}\right)_{2} \mathrm{CuCl}_{4}$ [cf. Fig. 1(d)].
The organic spacers are bilayers of organic ammonium placed tail to tail in neighboring layers. The ammonium heads of the organic cation hydrogen bond to the halogens in the inorganic sheet while the organic tails extend into the spacer with the van der Waals interaction between the tails of the successive layers [18].

Starting from the optimized crystal structures for the 3D compounds, the corresponding 2D counterparts were constructed by keeping the single layer of extended 2D anions $\left(\mathrm{CuCl}_{4}\right)^{2-}$ connected to the organic cation on both sides for charge neutrality and removing the rest from the cell as shown in Fig. 1. The derived two-dimensional structures were further optimized within the DFT framework with a choice of the generalized gradient approximation (GGA) of the exchange correlation together with the supplemented Hubbard $U$ correction $(\mathrm{GGA}+U)$ [19-23]. For details on the DFT computation, see the Supplemental Material (SM) [24].

The JT distortion of the $\mathrm{CuCl}_{6}$ octahedra, defined as $\frac{l-s}{l+s}$, and the buckling angle, which gives the deviation from the inplane $\mathrm{Cu}-\mathrm{Cl}-\mathrm{Cu} 180^{\circ}$ bond angle for the four 3D compounds, in comparison to the derived $2 \mathrm{D}$ counterparts are shown in Fig. 1(e). As can be seen, the structural parameters such as JT distortions and buckling angles change the maximum by about $7 \%$ between the $3 \mathrm{D}$ and $2 \mathrm{D}$ variants, suggesting the structural properties to be affected only moderately in moving from $3 \mathrm{D}$ to $2 \mathrm{D}$.

Cleavage energy. A possible means to derive the abovediscussed 2D counterparts from their existing 3D variants is mechanical exfoliation. However, for the successful exfoliation of various $\left(R-\mathrm{NH}_{3}\right)_{2} \mathrm{CuCl}_{4}(R=\mathrm{EA}, \mathrm{CPMA}$, PEA, Naphtha-4-MA) species to 2D structures, an energy barrier needs to be overcome, known as the cleavage energy. The cleavage energy $\left(E_{\mathrm{cl}}\right)$ for the present case can be defined as the energy required to generate two (top and bottom) surfaces by cleaving the bulk layered structure along the $\mathrm{CuCl}$ plane $[25,26], E_{\mathrm{cl}}=2 \frac{\left(E_{\text {slab }}-E_{\text {bulk }}\right)}{2 A}$, where $E_{\text {slab }}$ is the total energy of the cleaved system with two exposed surfaces (while the individual layers are sufficiently far to be out of the range of dispersive interactions) and $E_{\text {bulk }}$ is the total energy of the same in the bulk configuration, with $A$ being the surface area. To this end, considering the four $3 \mathrm{D}$ structures, the interlayer separations $(d)$ were gradually increased in the range $0.2-10 \AA$ (cf. the insets in Fig. 2) and the surface area-normalized total energy differences between the layer-separated and bulk models were calculated, as shown in Fig. 2. It can be readily observed that, for all considered $A_{2} \mathrm{CuCl}_{4}$ structures, the energy increases with $d$ and rapidly converges to $E_{\mathrm{cl}}$, with values $0.161,0.151,0.191$, and $0.176 \mathrm{~J} / \mathrm{m}^{2}$ for $\left(\mathrm{EA}-\mathrm{NH}_{3}\right)_{2} \mathrm{CuCl}_{4},\left(\mathrm{CPMA}-\mathrm{NH}_{3}\right)_{2} \mathrm{CuCl}_{4}$, $\left(\mathrm{PEA}-\mathrm{NH}_{3}\right)_{2} \mathrm{CuCl}_{4}$, and (Naphtha-4-MA-NH$)_{2} \mathrm{CuCl}_{4}$, respectively. Compared to the cleavage energy of graphite, estimated to be $0.37 \mathrm{~J} / \mathrm{m}^{2}$ [27], these cleavage energies are a factor of 2-2.5 smaller, indicating these layered inorganicorganic perovskites possess a significantly weaker interlayer coupling compared to graphite. This suggests, in line with the trend previously observed for $(\mathrm{PEA})_{2} \mathrm{PbI}_{4}$ [28], these materials are highly promising towards exfoliation down to atomically thin layers. The dynamical stability of the $2 \mathrm{D}$ structures has been validated through calculated phonon spectra, as presented in the SM. 


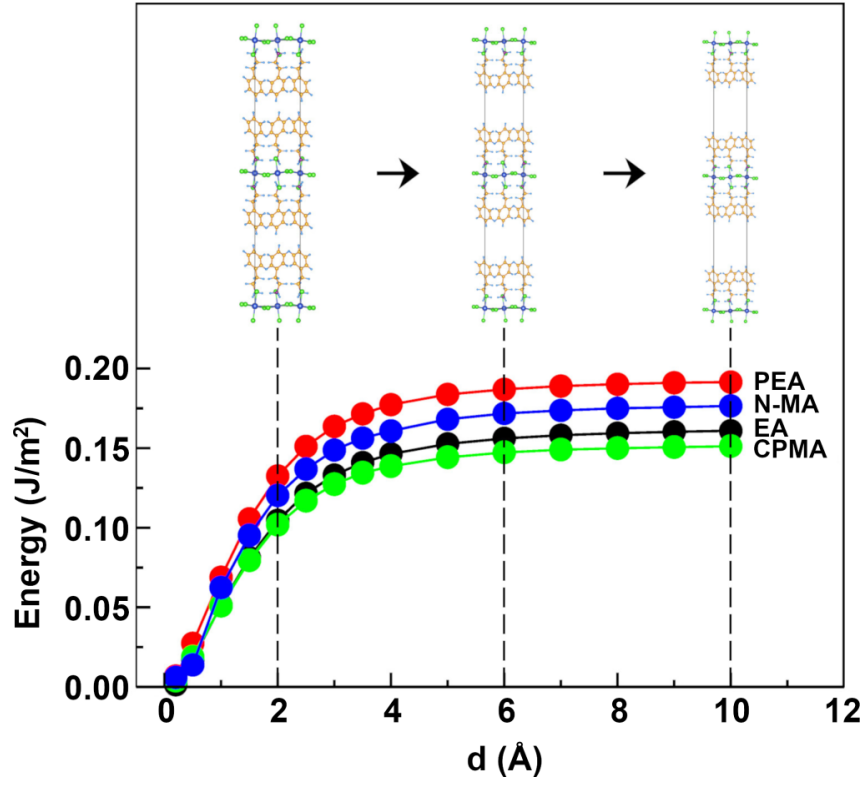

FIG. 2. Calculated energy cost in separating the layers as as function of interlayer distance $(d)$ for $\left(R-\mathrm{NH}_{3}\right)_{2} \mathrm{CuCl}_{4}(R=\mathrm{EA}$, CPMA, PEA, Naptha-4-MA). The converged value of the energy cost at large $d$ provides the estimate of cleavage energy. The inset shows the pictorial description of cleavage energy calculation.

Magnetic exchange and anisotropy energy. To estimate the magnetic exchanges, we considered four ordered spin states for the magnetic $\mathrm{Cu}^{2+}$ ion, viz., ferromagnetic (FM), A-type antiferromagnetic $(A-\mathrm{AFM})$ with $\mathrm{Cu}$ spins aligned parallely within the plane and antiparallely out of the plane, $C$-type antiferromagnetic $(C$-AFM) with spins aligned antiparallely in the plane and parallely out of plane, and $G$-type antiferromagnetic ( $G$-AFM) with $\mathrm{Cu}$ spins aligned antiparallely in all directions. The computed magnetic moments were found to be about $0.64 \mu_{B}-0.66 \mu_{B}$ at each $\mathrm{Cu}$ site, with a total moment of $4 \mu_{B}$ in the cell in the FM configuration, corresponding to four $\mathrm{Cu}$ ions in the cell. A large part of the moment was found at the $\mathrm{Cl}$ sites, suggesting strong $\mathrm{Cu}-\mathrm{Cl}$ covalency. The quantitative estimates of the effective magnetic exchange couplings $J_{i j}$ 's between two $\mathrm{Cu}$ centers at sites $i$ and $j$ were obtained by mapping the GGA $+U$ energies for the four ordered spin states (FM, $A$-AFM, $C$-AFM, and $G$-AFM) onto the spin model with $S=1 / 2$, as appropriate for the $\mathrm{Cu}^{2+}$ ion having a $3 d^{9}$ electron configuration; $H=\sum_{\langle i j\rangle} J_{i j} \mathbf{S}_{i} \cdot \mathbf{S}_{j}\left(\mathbf{S}_{i}\right.$ is the spin operator at the site $i, J<0$ implies FM exchange, while $J>0$ implies AFM exchange).

For the 3D $\left(R-\mathrm{NH}_{3}\right)_{2} \mathrm{CuCl}_{4}$ compounds, the range of the magnetic exchanges between the $\mathrm{Cu}^{2+}$ ions was limited to four nearest-neighbor $\left(J_{\text {in }}\right)$ interactions within the square plane of $\mathrm{CuCl}$, and eight out-of-plane $\left(J_{o}\right)$ interactions. The variation of $J_{\text {in }}$ and $J_{o}$ within the four studied systems is shown in Fig. 3(a). The large interlayer separation between the $\mathrm{CuCl}_{4}{ }^{2-}$ layers causes the out-of-plane magnetic exchange constant $J_{o}$ to be small $(<0.1 \mathrm{meV})$, and of an antiferromagnetic nature in conformity with the stabilization of the $A$-AFM magnetic state, with the exception of (PEA- $\left.\mathrm{NH}_{3}\right)_{2} \mathrm{CuCl}_{4}$, which is found to be FM. $J_{\text {in }}$ in all cases was found to be ferromagnetic due to the antiferrodistortive arrangement (a)

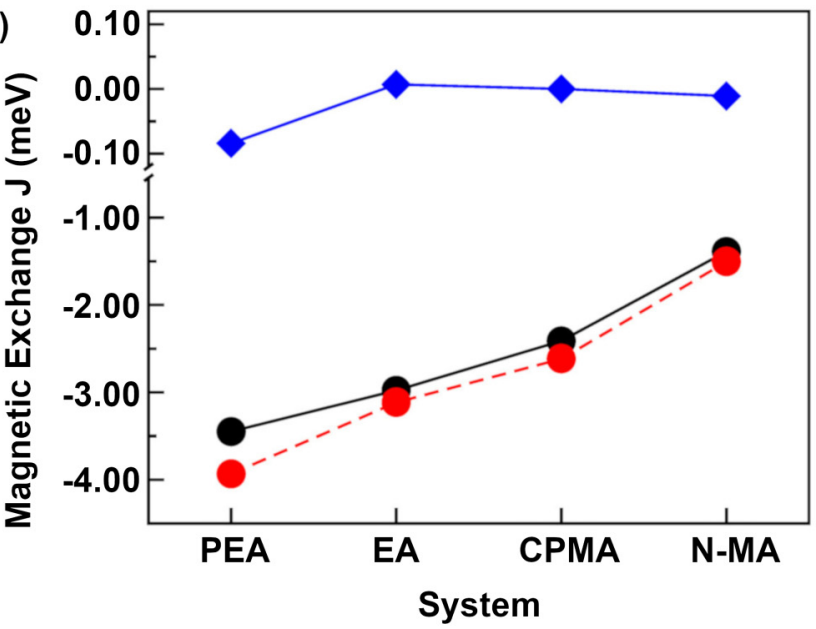

(b)

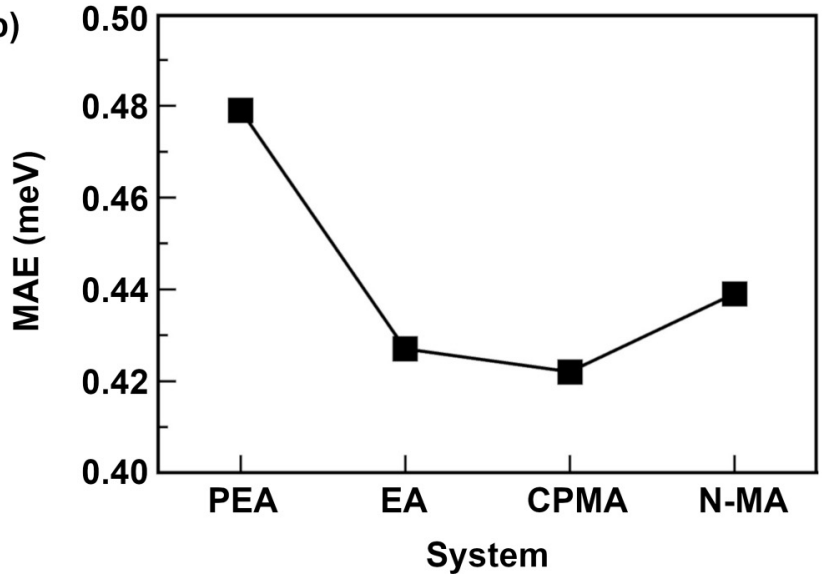

FIG. 3. (a) $J_{\text {in }}$ for $\left(\mathrm{EA}-\mathrm{NH}_{3}\right)_{2} \mathrm{CuCl}_{4},\left(\mathrm{CPMA}-\mathrm{NH}_{3}\right)_{2} \mathrm{CuCl}_{4}$, $\left(\mathrm{PEA}-\mathrm{NH}_{3}\right)_{2} \mathrm{CuCl}_{4}$, and (Naphtha-4-MA- $\left.\mathrm{NH}_{3}\right)_{2} \mathrm{CuCl}_{4}$ in bulk (black symbols) and in the corresponding 2D geometries (red symbols). Shown are also $J_{o}$ 's in blue symbols for the bulk structures. (b) Magnetic anisotropy energy (MAE) for the 2D structures.

of $\mathrm{CuCl}_{6}$ octahedra with the JT-active $\mathrm{Cu}^{2+}$ ion, supporting the FM interaction through a near $180^{\circ}$ superexchange path. Following the same procedure we further computed the magnetic exchanges for the 2D system, which has only in-plane magnetic exchanges. The calculated in-plane exchanges for the 2D systems are shown in Fig. 3(a) in comparison to that of 3D systems. We find that the in-plane ferromagnetic correlation is retained even in the $2 \mathrm{D}$ limit, and moving from $3 \mathrm{D}$ to $2 \mathrm{D}$ geometry, the in-plane ferromagnetic interaction gets further strengthened. See SM for the energies of different magnetic states.

Having established the presence of a ferromagnetic correlation, we next investigate the possibility of stabilizing the ferromagnetic long-range order within the $2 \mathrm{D}$ geometry of the $\left(R-\mathrm{NH}_{3}\right)_{2} \mathrm{CuCl}_{4}$ compounds. As mentioned in the Introduction, long-range magnetic order is completely suppressed in 2D spin-rotationally invariant systems. The breaking of spinrotational invariance can happen in several different ways, one of the most obvious ones being single-ion anisotropy (SIA). We thus performed DFT calculations including spinorbit coupling (SOC), essential to account for the magnetic 
anisotropy. GGA $+U+\mathrm{SOC}$ calculations gave rise to a large unquenched orbital moment of $0.1 \mu_{B}$ at the $\mathrm{Cu}$ site, contrary to the expected fully quenched orbital moment in the $d^{9}$ JT-active ion, arising due to a large covalency between $\mathrm{Cu}$ and $\mathrm{Cl}$. Calculations carried out choosing the magnetization axis to be out of plane and in plane showed in-plane orientation to be favored over the out-of-plane orientation with an energy gain of $0.4-0.5 \mathrm{meV}$ per unit cell [cf. Fig. 3(b)]. With two $\mathrm{Cu}$ ions in the unit cell of 2D geometry, this gives a MAE of $\approx 0.2 \mathrm{meV}$ per $\mathrm{Cu}$ ion, translating into an in-plane single-ion anisotropy constant of value $0.8-0.9 \mathrm{meV}$. These estimates are more than an order of magnitude larger, and opposite in sign compared to the estimated value of $0.02 \mathrm{meV}$ for 2D $\mathrm{Cr}_{2} \mathrm{Ge}_{2} \mathrm{Te}_{6}$ crystals [5], for which the magnetic longrange order was stabilized only upon applying a magnetic field [5].

In the following, we take up the finite-temperature magnetic properties of the proposed 2D hybrid perovskites within a model Hamiltonian study.

Solution of a generalized spin model. The spin Hamiltonian, describing the magnetic properties of the proposed 2D hybrid materials, contains a nearest-neighbor isotropic Heisenberg exchange term between the $\mathrm{Cu}$ spins in the $2 \mathrm{D}$ square lattice of the 2D hybrid structure, which is ferromagnetic in nature and is a SIA term [29]. As opposed to previous studies of the SIA of $S=1 / 2$ systems, where a classical spin model was employed [30,31], here we treat the spin-half operators quantum mechanically, and consider a generalized form of SIA. The spin Hamiltonian, defined on a two-dimensional square lattice, reads as follows,

$$
H=-|J| \sum_{\langle i, j\rangle} \mathbf{S}_{i} \cdot \mathbf{S}_{j}+\sum_{i, \alpha, \beta} A_{i \alpha \beta} S_{i \alpha} S_{i \beta} .
$$

$S_{i \alpha}$ denotes the $\alpha$ th component of the spin operator at the $i$ th site $(i=1, \ldots, N), \alpha=x, y, z$, with $A_{i \alpha \beta}$ being a complex matrix [32], defined at every site $i$.

We denote the generic coefficient of $A_{i \alpha \beta}$ by $p_{i \alpha \beta}+\mathrm{iq} q_{i \alpha \beta}$. Then the Hamiltonian can be rewritten, omitting the constant energy shift, as

$$
H=-|J| \sum_{\langle i, j\rangle} \mathbf{S}_{i} \cdot \mathbf{S}_{j}-2 \sum_{i} \mathbf{B}_{i} \cdot \mathbf{S}_{i},
$$

where $\mathbf{B}_{i}=q_{i y z} \hat{x}+q_{i z x} \hat{y}+q_{i x y} \hat{z}$ is an effective pseudomagnetic field on the Heisenberg model. The spectrum of the SIA term is given by $\pm 2 \sqrt{q_{i y z}^{2}+q_{i z x}^{2}+q_{i x y}^{2}}$. Here, the negative (positive) eigenvalue implies the spin is oriented parallel (antiparallel) to the direction of $\mathbf{B}$. Since in DFT we find that the spins prefer to lie in the plane, with an anisotropy energy $\Delta$, we set $q_{x y}=0$, making the $\mathbf{B}$ planar, reducing the $\mathrm{SU}(2)$ symmetry to $U(1)$. This leads to the relation $\Delta^{2} / 4=q_{i y z}^{2}+$ $q_{i z x}^{2}$ [33]. Then, within a quadratic Holstein-Primakoff approximation [34], the spin-wave dispersion is given as $\omega(\mathbf{k})=$ $\sum_{k_{x}, k_{y}}\left\{J\left(4-2\left[\cos \left(k_{x}\right)-\cos \left(k_{y}\right)\right]\right)+\Delta\right\} b_{k}^{\dagger} b_{k}$ where $b_{k}^{\dagger}$ is the magnon creation operator. The spectrum has a gap set by the anisotropy energy and hence can stabilize the FM state at finite temperatures, with $T_{c} \propto \Delta$. The exact spin-wave spectra $S(\omega)$, which can calculated using the Lanczos algorithm [35], as

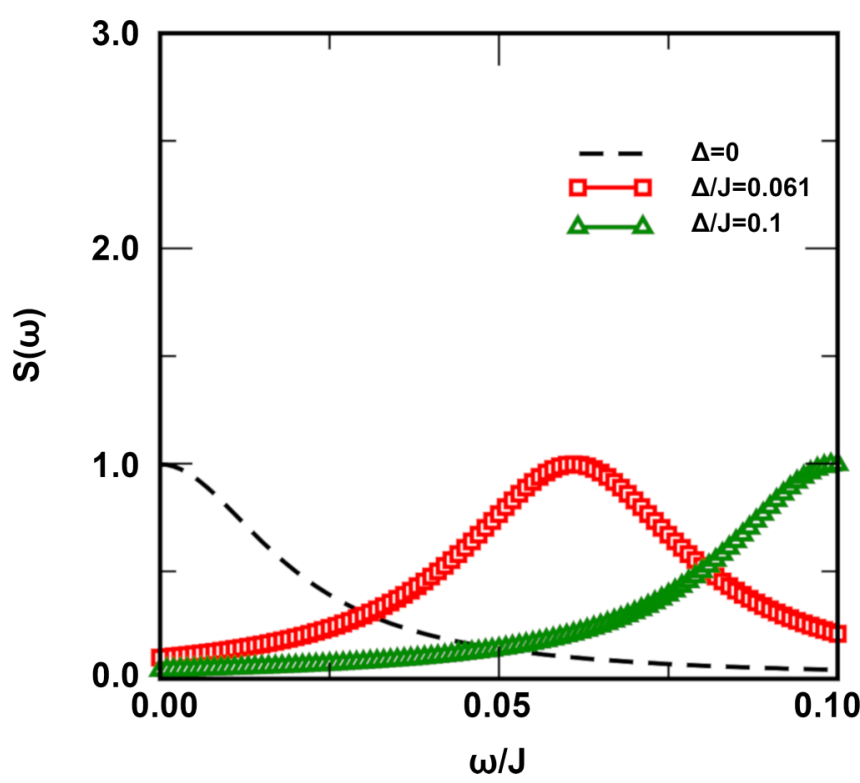

FIG. 4. The low-energy part of the spin-wave spectrum for different values of the anisotropy parameter $(\Delta / J)$, calculated using the Lanczos technique on a $4 \times 6$ lattice. The value of $\eta$ was set to 0.02 (see text for details).

used in the present study, is given by

$$
S(\omega)=\frac{1}{N} \sum_{i, \gamma}\left\langle\Psi_{G}\left|S_{i \gamma} \frac{1}{z \hat{I}-H+E_{G}} S_{i \gamma}\right| \Psi_{G}\right\rangle .
$$

$\left|\Psi_{G}\right\rangle$ and $E_{G}$ are the wave function and the energy in the FM ground state, respectively. $z=\omega+i \eta$, with $\eta$ being the broadening introduced, as is standard practice. Figure 4 shows $S(\omega)$ vs $\omega$ for three values of $\Delta / J$, including the case of zero anisotropy and the DFT estimated value of anisotropy. We see that the gap (the location of the peak of the curves measured from $\omega=0$ ) scales with $\Delta$. The spin-wave spectra relevant for the material-specific parameter $(\Delta / J=0.06)$ for $2 \mathrm{D} \mathrm{Cu}$ hybrids, given by DFT (shown in red, squares in the figure), gives rise to an estimated $T_{c}$ of a few degrees Kelvin, which, though low, is finite.

Conclusions. Motivated by the recent excitement in 2D ferromagnetic materials, we explored such possibilities within the family of layered inorganic-organic hybrid perovskites. Our computational exercise on Cu-based systems revealed that exfoliation of these layered materials to $2 \mathrm{D}$ monolayers is highly probable, given the fact that the computed cleavage energies turned out to be a factor of 2-3 smaller than that of graphite. The ferromagnetic nature of in-plane $\mathrm{Cu}$ $\mathrm{Cu}$ exchanges, driven by the antiferrodistortive arrangement of the JT-active $\mathrm{Cu}$ ions in the square coordination of $\mathrm{Cl}$ ions, remains intact in moving from bulk to $2 \mathrm{D}$ geometries. Calculated single-ion anisotropy showed it to be an order of magnitude higher than that of $\mathrm{Cr}_{2} \mathrm{Ge}_{2} \mathrm{Te}_{6}$ [5]. The anisotropy for hybrid $\mathrm{Cu}$ systems was found to be of an easy-plane type rather than an easy-axis type as found for $\mathrm{Cr}_{2} \mathrm{Ge}_{2} \mathrm{Te}_{6}$. The finite-temperature ordering of such an easyplane, spin- $1 / 2 \mathrm{Cu}$ spin was shown to be achievable within the formulation of a spin Hamiltonian with a generalized form of anisotropy, with the off-diagonal terms of the anisotropy 
matrix being responsible for driving the ordering at finite temperature.

Our study opens up the search space for 2D ferromagnetic materials to the interesting class of layered hybrid perovskites. For example, it will be worth extending the study to layered hybrid perovskites with larger spin, such as $\mathrm{Cr}$, for which a study is currently underway [36]. From the point of view of a model study, we would further like to explore the properties of the spin Hamiltonian containing a generalized form of the anisotropy term, which may give rise to a nontrivial spin texture and topological properties, the potential of which is yet to be explored.

Acknowledgments. T.S.-D. thanks the Department of Science and Technology, India for support through the Thematic Unit of Excellence. D.S. thanks University Grants Commission, Government of India, for providing financial support under the D. S. Kothari Postdoctoral Fellowship scheme. T.S.-D. gratefully acknowledges discussions with Thom Palstra for bringing these interesting compounds to our attention.
[1] K. S. Novoselov, D. Jiang, F. Schedin, T. J. Booth, V. V. Khotkevich, S. V. Morozov, and A. K. Geim, Proc. Natl. Acad. Sci. USA 102, 10451 (2005).

[2] K. S. Novoselov, A. Mishchenko, A. Carvalho, and A. H. Castro Neto, Science 353, aac9439 (2016).

[3] M. Gibertini, M. Koperski, A. F. Morpurgo, and K. S. Novoselov, Nat. Nanotechnol. 14, 408 (2019).

[4] N. D. Mermin and H. Wagner, Phys. Rev. Lett. 17, 1133 (1966).

[5] C. Gong, L. Li, Z. Li, H. Ji, A. Stern, Y. Xia, T. Cao, W. Bao, C. Wang, Y. Wang, Z. Q. Qiu, R. J. Cava, S. G. Louie, J. Xia, and X. Zhang, Nature (London) 546, 265 (2017).

[6] B. Huang, G. Clark, E. Navarro-Moratalla, D. R. Klein, R. Cheng, K. L. Seyler, D. Zhong, E. Schmidgall, M. A. McGuire, D. H. Cobden, W. Yao, D. Xiao, P. Jarillo-Herrero, and X. Xu, Nature (London) 546, 270 (2017).

[7] B. Huang, G. Clark, D. R. Klein, D. MacNeill, E. NavarroMoratalla, K. L. Seyler, N. Wilson, M. A. McGuire, D. H. Cobden, D. Xiao, W. Yao, P. Jarillo-Herrero, and X. Xu, Nat. Nanotechnol. 13, 544 (2018).

[8] K. L. Seyler, D. Zhong, D. R. Klein, S. Gao, X. Zhang, B. Huang, E. Navarro-Moratalla, L. Yang, D. H. Cobden, M. A. McGuire, W. Yao, D. Xiao, P. Jarillo-Herrero, and X. Xu, Nat. Phys. 14, 277 (2018).

[9] Editorial, Nat. Nanotechnol. 13, 269 (2018).

[10] M. Bonilla, S. Kolekar, Y. Ma, H. Coy Diaz, V. Kalappattil, R. Das, T. Eggers, H. R. Gutierrez, M.-H. Phan, and M. Batzill, Nat. Nanotechnol. 13, 289 (2018).

[11] D. B. Mitzi, in Progress in Inorganic Chemistry, edited by K. D. Karlin (Wiley, Hoboken, NJ, 1999), Vol. 9, pp. 1-121.

[12] T. Oku, J. Nomura, A. Suzuki, H. Tanaka, S. Fukunishi, S. Minami, and S. Tsukada, Int. J. Photoenergy 2018, 8654963 (2018).

[13] M. M. Lee, J. Teuscher, T. Miyasaka, T. N. Murakami, and H. J. Snaith, Science 338, 643 (2012).

[14] J. Burschka, N. Pellet, S.-J. Moon, R. Humphry-Baker, P. Gao, M. K. Nazeeruddin, and M. Grätzel, Nature (London) 499, 316 (2013).

[15] A. Polyakov, Soluble multiferroic hybrids: Controlling the electronic interactions, Ph.D. thesis, University of Groningen, 2015.

[16] L. Dou, A. B. Wong, Y. Yu, M. Lai, N. Kornienko, S. W. Eaton, A. Fu, C. G. Bischak, J. Ma, T. Ding, N. S. Ginsberg, L.-W. Wang, A. P. Alivisatos, and P. Yang, Science 349, 1518 (2015).

[17] A. O. Polyakov, A. H. Arkenbout, J. Baas, G. R. Blake, A. Meetsma, A. Caretta, P. H. M. van Loosdrecht, and T. T. M. Palstra, Chem. Mater. 24, 133 (2012).

[18] The details of the hydrogen bonding depend on the orientation, and conformation of the organic molecules. The
$\mathrm{N}-\mathrm{H} \cdots X$ hydrogen bonding either forms two in-plane bridgings, one out-of-plane terminal halide or two terminal, one bridging halide. The former is known as the bridging halide configuration, (CPMA- $\left.\mathrm{NH}_{3}\right)_{2} \mathrm{CuCl}_{4}$, and the latter is known as the terminal halide configuration. Out of the four compounds considered by us, the first three, $\left(\mathrm{EA}-\mathrm{NH}_{3}\right)_{2} \mathrm{CuCl}_{4}$, (PEA- $\left.\mathrm{NH}_{3}\right)_{2} \mathrm{CuCl}_{4}$, show a terminal halide configuration, and the last one, (Naphtha-4-MA- $\left.\mathrm{NH}_{3}\right)_{2} \mathrm{CuCl}_{4}$ shows a bridge halide configuration. Consideration of four compounds thus provides a comparison of the two possible configurations.

[19] G. Kresse and J. Furthmüller, Comput. Mater. Sci. 6, 15 (1996).

[20] G. Kresse and D. Joubert, Phys. Rev. B 59, 1758 (1999).

[21] J. P. Perdew, K. Burke, and M. Ernzerhof, Phys. Rev. Lett. 77, 3865 (1996).

[22] S. L. Dudarev, G. A. Botton, S. Y. Savrasov, C. J. Humphreys, and A. P. Sutton, Phys. Rev. B 57, 1505 (1998).

[23] S. Grimme, J. Antony, S. Ehrlich, and H. Krieg, J. Chem. Phys. 132, 154104 (2010).

[24] See Supplemental Material at http://link.aps.org/supplemental/ 10.1103/PhysRevResearch.1.032034 for details of the DFT basis set, choice of exchange correlation, $k$-point integration, structural optimization, cleavage energy calculations, bulk structural details, energies of different magnetic configurations, and phonon spectra.

[25] R. Benedek and M. M. Thackeray, Phys. Rev. B 83, 195439 (2011).

[26] D. Santos-Carballal, A. Roldan, R. Grau-Crespo, and N. H. de Leeuw, Phys. Chem. Chem. Phys. 16, 21082 (2014).

[27] W. Wang, S. Dai, X. Li, J. Yang, D. J. Srolovitz, and Q. Zheng, Nat. Commun. 6, 7853 (2015).

[28] J. Gebhardt, Y. Kim, and A. M. Rappe, J. Phys. Chem. C 121, 6569 (2017).

[29] From the density functional calculations we find that the nearest-neighbor exchanges are isotropic and dipolar interactions are negligible. Thus, the single-ion anisotropy term is the natural choice for investigating the stability of the FM state at a finite temperature, particularly because the orbital angular moment is unquenched.

[30] H. Xiang, C. Lee, H. Joo Koo, X. Gong, and M. Hwan Whangbo, Dalton Trans. 42, 823 (2013).

[31] J. Liu, H.-J. Koo, H. Xiang, R. K. Kremer, and M.-H. Whangbo, J. Chem. Phys. 141, 124113 (2014).

[32] We do not attempt to calculate the microscopic requirements for this condition. Our model is purely a phenomenological proposal for stabilizing FM at a finite temperature arising out of single-ion anisotropy in two dimensions, where the half-spin operators are treated quantum mechanically. However, we note 
that for $S=1 / 2$, the general structure of the $3 \times 3$ complex matrix for $A_{\alpha, \beta}$, with complex off-diagonal and real diagonal terms, can accommodate contributions from terms that might arise at any order of perturbation in the spin-orbit coupling term. The reality of the diagonal terms is required for Hermiticity. Further, the real diagonal terms of the SIA matrix can be shown to give a constant shift to the Heisenberg term. A similar quantum mechanical model can also in principle stabilize FM for $S>1 / 2$ in two dimensions. These investigations will be reported separately.

[33] We observe that, in the $x y$ plane, the anisotropy energy can be satisfied by any choice of $q_{i y z}$ and $q_{i z x}$, as long as they lie on the circle defined by the above equation. Further, the eigenvectors corresponding to the two eigenvalues are $1 / \sqrt{2}\left( \pm e^{i \theta}, 1\right)$, with $\theta=\tan ^{-1}\left(q_{z x} / q_{y z}\right)$. Thus, once a choice is made for $q_{i y z}$ and $q_{i z x}$, the $\mathbf{B}$ vector breaks the in-plane $U(1)$ symmetry and provides an easy axis, thereby stabilizing the FM state. Since any $q_{i y z}$ and $q_{i z x}$ are valid, consistent with the above equation, we choose $q_{i z x}=0$. Thus, $\mathbf{B}=q_{i y z} \hat{x}=-(|\Delta| / 2) \hat{x}$.

[34] T. Holstein and H. Primakoff, Phys. Rev. 58, 1098 (1940).

[35] C. Lanczos, J. Res. Natl. Bur. Stand. US 45, 255 (1950).

[36] D. Sen, D. Nafday, N. Kaushal, A. Mukherjee, and T. SahaDasgupta (unpublished). 